\title{
A Historical Review and Current Perspective on the Intervertebral Disc Prosthesis
}

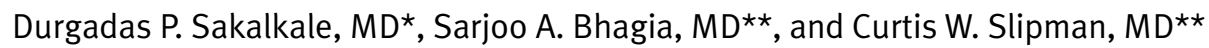

Spinal fusion for degenerative disc disease has been associated with a variety of side effects, including increased morbidity, infection, failed back syndrome, pseudoarthrosis, and acceleration of degenerative changes in adjacent intervertebral discs and facet joints. Based on the experience of arthroplasty of hip and other joints, there has been an escalating in research emphasizing the design and development of an artificial disc prosthesis. However, these artificial discs have not been very successful when compared with hip or knee replacements.

Based on clinical and anatomical data, multiple authors have postulated that the degenerative process of intervertebral disc evolves through three stages; dysfunction, instability and stabilization, with relatively distinct clinical and radiological findings. Even though magnetic resonance imaging is considered as the primary diagnostic tool for degenerative disease, it is unable to reliably ascertain which disc level is responsible for generating axial pain symptoms. Consequently, discography is the most precise test to localize the level of pathology.

Multiple design criteria have been proposed for an ideal intervertebral prosthesis which included endurance, materials behavior, geometry, kinematics, dynamics, motion constraints, fixation to bones and safety. The development of artifical disc technology has culminated into two types of disc replacements, namely total disc replacement and a nucleus pulposus replacement. In addition, four prosthetic models have been proposed which include hydraulic, elastic, composite, and mechanical. Clinical outcomes of total disc replacement and nucleus replacement have been variable.

This review describes natural history of disc disease and the diagnostic process, anatomical and biomechanical considerations, design criteria for an ideal intervertebral prosthesis, evolution of artificial disc prosthesis, clinical outcomes of the total disc and nucleus replacement, and prospects and meridians for future research.

Keywords: Intervertebral disc, degenerative disc disease, disc prosthesis, disc replacement
Spinal Fusion has been considered the primary surgical treatment for degenerative disc disease following failure of other forms of treatment. Unfortunately, it has been associated with variety of side effects and complications including increased morbidity, infection, failed back syndrome and pseudarthrosis (1-2). Moreover, it has been shown to accelerate degenerative changes in adjacent intervertebral discs and facet joints secondary to increased stresses and mobility in these segments (3-5). Arthrodesis or resection arthroplasty used to be the option for treatment of severe degenerative arthritis of hip joints until the advent of total hip arthroplasties(6). Following the pioneering work of Sir John Charnley (6)

From *The Department of Physical Medicine and Rehabilitation, Rusk Institute of Rehabilitation Medicine, New York, NY and ${ }^{* *}$ The Department of Rehabilitation Medicine, University of Pennsylvania Medical Center, Philadelphia, PA. Address Correspondence: Curtis W. Slipman, MD, Hospital of the University of Pennsylvania, 3400 Spruce Street, Ground White Building, Philadephia, PA 19104. Email: slipman@mail.med.upenn.edu.

There has been no support in the form of grants, equipment, or drugs. in the sixties, the last three decades have witnessed a rapid evolution of total joint technology (7-8). As well, an understanding of the intricacies of the bone implant interface has been garnered (9).

Analogically, Fernstorm (10) deserves the distinction of being the first to use stainless steel metal balls to replace degenerative discs. Subsequently, there has been an escalating interest in research emphasizing the design and development of an artificial disc prosthesis. These artificial discs have not been very successful when compared with hip or knee replacements for several reasons; the complexity of disc structure and function, higher risk involved if the device fails and relatively lesser impairment to patient's function with single level disc fusion as compared with hip or knee fusion.

\section{Natural History of Disc Disease and The Diagnostic Process}

In their extensive study of degenerative disc disease, based on clinical and anatomical data, authors (11-15) postulated that the degenerative process of intervertebral disc evolves through three stages. dysfunction, instability and stabiliza- tion, with relatively distinct clinical and radiological findings. Histological studies of Miller et al(15) further substantiate the existence of these three stages. The dysfunction stage typically presents in the 15 and 45 years old age group. It is characterized by circumferential and radial tears in the disc annulus and localized synovitis of the facet joints. The instability stage, found in 35 to 70 years old patients, consists of various processes such as internal disruption of the disc, progressive disc resorption, degeneration of the facet joints with capsular laxity, subluxation, and joint erosion. The final stage of stabilization, which is rarely seen in patients younger than 60 years, evolves from the progressive development of hypertrophic bone about the disc and facet joints leading to segmental stiffening or frank ankylosis. Disc herniation is a manifestation that presents in the first two stages, while spinal stenosis is associated with late second and final stages of disc disease (12).

Magnetic resonance imaging is the primary diagnostic tool when working up symptoms of degenerative disc disease (16-18). Unfortunately, it is unable to reliably ascertain which disc level is respon- 
sible for generating axial pain symptoms. First described by Lindblom (19) in 1948, discography is the most precise test to localize the level of pathology. With its ability to diagnose the exact level of symptoms, discography has been advocated as the diagnostic pre-requisite when contemplating discectomy or spinal fusion (20-21).

\section{Anatomical and Biomechanical Considerations}

The natural intervetebral disc has anatomical and biomechanical properties, which serve an intricate role in stability and flexibility of spine. It consists of a nucleus, annulus and two end plates, which impart synchronizing biomechanical features. The annulus fibrosus has a multilayered structure made up of wellorganized collagen fibers running in opposite directions in adjacent layers at approximately 30 degrees to horizontal plane and provides high tensile modulus. The nucleus, which is enclosed within the annulus and endplates, is composed of the hydrophilic polymer, glycosaminoglycan. The nucleus provides compressive strength by keeping the annulus inflated. The endplates provide stable anchorage to the layers of annulus fibrosus thereby preventing excessive torsional and translational movements (22). Along with the facet joints it is responsible for absorbing all compressive loads directed to axial skeleton. Combined rotation and bending leads to stresses in the disc with tensile, compressive and shear components (22). Since each functional spinal unit is composed of three distinct anatomical structures, each with unique mechanical properties, the biomechanics and physiology of disc is much more complex than the hip or knee joint. The paucity of overall literature on artificial discs and the marginal success rates reported underscore the complexity of this structure.

An ideal intervertebral disc should restore optimal anatomical and biomechanical function of the functional spinal unit. The purpose of the prosthetic device is to attain normal disc height and maintain maximal intersegmental flexibility to physiological loads. The disc prosthesis should simulate typical discal kinematic properties to restore normal biomechanics.

\section{Design Criteria for an Ideal INTERVERTEBRAL PROSTHESIS}

Hedman et al (23) have proposed design criteria for an ideal intervertebral disc prosthesis including endurance, materials behavior, geometry, kinematics, dynamics, motion constraints, fixation to bones and safety. Considering the morbidity of spinal surgery, an ideal device should be able to last for life and should be tested for withstanding a greater number of spinal loading cycles than estimated for the expected longevity of the patient. An ideal material would have biocompatibility with greater resistance against wear and galvanic corrosion. The geometry of the artificial disc should reproduce that of the normal disc including disc height. The kinematics of the artificial disc should simulate the kinematics of the level of replacement. For example, a typical L4-5 intervertebral joint allows 13 degrees of flexion, 3 degrees of extension, 3 degrees of lateral bending and 1 degree of axial rotation (26). The locus of axes for sagittal rotation is located in the posterior region of the disc space (27). Reproducing the position of the axes of motion within the artificial disc is critical for restoration of the multiplanar motion of the natural intervetebral disc. The disc prosthesis should duplicate the stiffness of the natural disc in all three planes of rotation (23). An ideal prosthesis should impose the same constraints to motion as the natural joint provides. An overconstrained prosthesis would behave more like a fusion, whereas an underconstrained joint would fail to provide optimal stability. Stable and durable fixation of the bone-prosthesis interface is key to the long-term stability of the artificial joint. Currently, resolution of this issue represents the critical limiting factor impeding routine clinical use of a disc prosthesis. Advances in biologic fixation such as the biologic ingrowth may be able to solve this enormous concern. Destructive testing of the artificial joint should be performed prior to clinical trials to ensure that failure of the different components of the prosthesis will not transpire leading to damage to the surrounding nervous and vascular tissues (23). Animal model studies involving monkeys and sheep have expanded our understanding of the biomechanical capability and biocompatibility of the artificial disc prosthesis $(24,25)$. Unfortunately, the information obtained from such in- quiry may not be generalizable to human. In particular, the animal model lacks important biomechanical features of the human spine, such as axial loading, and longitudinal assessment of the longevity of the prosthesis is nearly impossible.

\section{Evolution of Artificial Disc Prostheses}

The development of artificial disc technology has culminated into two types of disc replacements. total disc replacement and nucleus pulposus replacement (28). Four prosthetic models have been proposed which include hydraulic, elastic, composite and mechanical (29).

\section{Hydraulic}

While Fernstrom's (10) pioneering trials used steel ball to replace the nucleus, Bao was the first to use a hydrogel material for a true nucleus prosthesis (17). The primary objective of the nuclear prosthesis is to inflate or expand the annulus and share the loads across the annulus (29). It has several theoretical advantages over a total disc prosthesis including preservation of natural annulus fibrosus, endplates and their function. As well, the surgical procedure is relatively uncomplicated to perform. While the advantages of this prosthesis are evident, its potential applications are limited because viable candidates require an essentially intact annulus. Other materials including polyurethane (Fassio and Ginestie) (29) and woven polymer fibers have been used in the past, albeit unsuccessfully. Complications experienced with this model include herniation of polyurethane and endplate fracture.

\section{Elastic Model}

This involves total disc replacement using a composite polymer matrix that would replicate normal annular structure. Steffe (31) used polyolefin rubber in six cases (Acroflex), but observed translation of the implant with slight or no rotation resulting in polymer shearing. Lee et al (32) proposed using a thermoplastic composite consisting of polysiloloxane reinforced with polyurethane fibers, as mechanism to increase axial and torsional strength. The major limitation of this model is the lack of optimal anchorage to the end plates to prevent translation during application of physiologic loading. As previously mentioned, the importance of this matter cannot be overemphasized. 


\section{Composite Model}

Edeland $(33,34)$ proposed using a composite viscohydraulic concept that would create an intrinsic preloading of the annular polymer, redefine the instantaneous axes of rotation and reduce stresses at the bone implant interfaces. To date, no clinical evidence is available to demonstrate the durability of the hydraulic systems or biomechanics of viscoelastic-metal interfaces.

\section{Mechanical Models}

These are artificial discs that employ a segmented joint model. Fixation occurs at the end plates. Mobility is effected with a pivot or ball type linkage (29). Only one these models have been subjected to clinical trials with promising outcomes. The SB Charite III Prosthesis (Fig 1), developed by Buttner-Janz and colleagues, is a modular system (33-37). It consists of two end plates of cobalt-chromium alloy and a polyethylene sliding core that reproduces near normal segment mobility. This design enables14 degrees of flexion/ extension. The end plates are available in two geometric configurations; parallel and oblique. The parallel design is used to replace segments above L5-S1 whereas the oblique model is designed for the anatomy of L5-S1 intervertebral space. The height of the polyethylene core changes from $7.5 \mathrm{~mm}$ to $11.5 \mathrm{~mm}$ to allow for op-

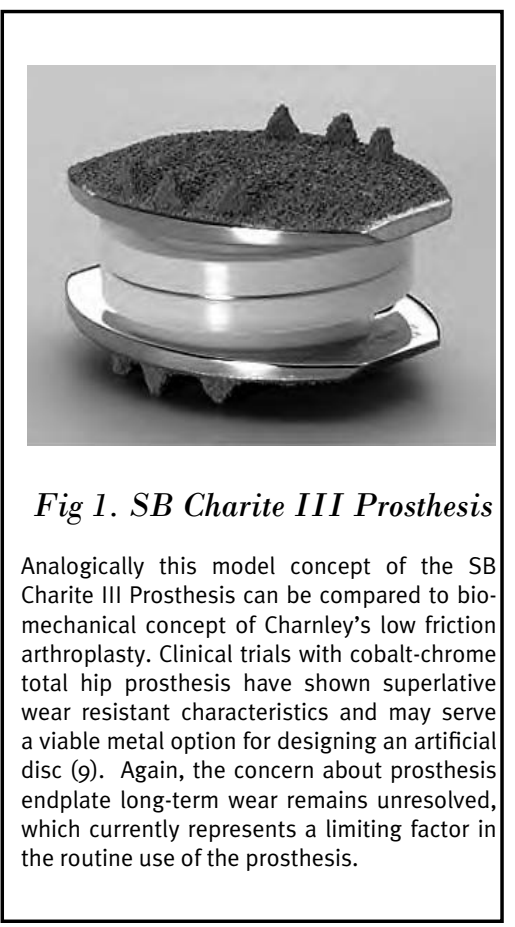

timum restoration of disc height.

\section{Clinical Outcomes of the Total Disc REPLACEMENT}

Pioneering work of Fernstrom (10) led to the first ever clinical trial of an artificial disc prosthesis in 1966. The clinical results of his ball bearing prosthesis were largely unsuccessful following subsidence into the adjacent vertebral bodies. Significant loss of disc height in $88 \%$ and a $40 \%$ incidence of residual low back pain were observed. In 1993, Enker et al (39) reported six cases of intervertebral disc replacement using the Acroflex prosthesis, comprising of a rubber core vulcanized to titanium end plates, with a minimum three year follow up. Four of the six patients had degenerative disc disease adjacent to a prior fusion, one had multilevel disc degeneration, and one had disc resorption. Four of the six patients had a satisfactory outcome. Translation of the prosthesis with fracture of the rubber core occurred in one patient at one year follow up requiring the performance of an interbody fusion. One patient had no relief of preoperative symptoms following the artificial disc replacement.

The SB Charite III Prosthesis (3538 ) is the most extensively used prosthesis with clinical trials held in Europe. In their multicenter retrospective study, Griffith et al (36) reported upon the use of 139 prostheses in 93 patients. This population had an average age of 43 years and mean follow up was $11.5 \pm 8.4$ months. The predominant pre-operative diagnosis was degenerative disc disease at the L4-5 or L5S1 level in $87 \%$ of patients. There was a significant improvement in pain relief of leg and back pain with improvement of the former due to surgical decompression rather than the direct effect of the prosthesis. Neurological weakness was present in $23 \%$ of the cases preoperatively. At follow up, $81 \%$ had resolution of neurological deficit. Complications observed included device failure, migration and dislocation in 6 of the $93(6.5 \%)$ cases.

\section{Clinical Outcomes of Nucleus REPLACEMENT}

There has not been any published report of clinical trial of the nucleus replacement prosthesis. At the first Annual North American Artificial Disc Summit in October 1999, Raymedica reported 101 patients with nucleus replacement with complication of implant extrusion in
17 patients (28).

Clinical trials performed have been primarily restricted to the use of the artificial disc prosthesis for lumbar degenerative disc disease. There is only one report of cervical disc replacement and dismal results were noted. Pointillart et al (40) reported failure of the cervical disc prosthesis in 8 of the 10 patients.

\section{Prospects and Meridians for future RESEARCH}

Given the well known limitations of spinal fusion and the advent of artificial disc technology further research oriented towards developing biomechanically superior designs with greater biocompatibility will inevitably continue (27). Kotani et al (25) have reported upon the use of a three dimensional bioactive fabric for artificial disc replacement in an effort to improve stability at the bone implant interface. A sheep spine model was used to establish as potential for future clinical application. As previously mentioned animal model studies do not adequately replicate the human spine; therefore research is requisite to create a laboratory model which would reproduce biomechanical forces typically generated in the human spine. Similarly, research into developing cervical disc prostheses with optimal biomechanical features will ensue. To date, none of the available intervertebral disc prostheses has been approved by FDA for clinical use in United States. However, there are on going FDA approved clinical trials of the SB Charite III prosthesis in various centers across North America.

\section{Author Affiliation: \\ Durgadas P. Sakalkale, MD,}

PGY III Resident, Department of Physical Medicine and Rehabilitation Medicine, Rusk Institute of Rehabilitation Medicine, New York, NY

\section{Sarjoo A. Bhagia, MD}

Fellow, Penn Spine Center, Department of Rehabilitation Medicine, University of Pennsylvania Medical Center, Pennsylvania, PA

\section{Curtis Slipman, MD}

Associate Professor, Director of Penn Spine Center, Department of Rehabilitation Medicine, University of Pennsylvania Medical Center, Pennsylvania, PA Email: slipman@ mail.med.upenn.edu 


\section{REFERENCES}

1. Stambough JL. Lumbosacral instrumented fusion: analysis of 124 consecutive cases. J Spinal Disord 1999; 12:1-9.

2. Brown CA, Eismont FJ. Complications in spinal fusion. Orthop Clin North Am 1998;29:679-699

3. Kumar MN, Jacquot F, Hall H. Long-term follow-up of functional outcomes and radiographic changes at adjacent levels following lumbar spine fusion for degenerative disc disease. Eur Spine J 2001;10:309-313.

4. Phillips FM, Reuben J, Wetzel FT. Intervertebral disc degeneration adjacent to a lumbar fusion. An experimental rabbit model. Bone J Surg [Br] 2002; 84:289-294.

5. Chen CS, Cheng CK, Lu CL et a1. Stress analysis of the disc adjacent to interbody fusion in lumbar spine. Med Eng Physic 2001; 23:483-491.

6. Charnley J. Total hip replacement by low-friction arthroplasty. Clinical Ortho 1970;72:7-21

7. Callaghan JJ, Albright JC, Goetz DD et al. Charnley total hip arthroplasty with cement. Minimum twenty-five-year followup. J Bone J Surg 2000; 84:487-497

8. Coventry MB. Lessons learned in 30 years of total hip arthroplasty. Journal Review. Clin Orthop 1992; 274:22-29.

9. Gorab RS, Covino BM, Borden LS. The rationale for cementless revision total hip replacement with contemporary technology. [Review of Cases] Orthop Clin North Am 1993; 24:627-633.

10. Fernstrom U. Arthroplasty with intercorporal endoprothesis in herniated disc and in painful disc. Acta Chir Scand 1966;357: 154-159.

11. Wedge J. The natural history of spinal degeneration. In Kirkaldy-Willis WH (ed). Managing low back pain. Churchill Livingstone, New York, 1983; pp 75-90.

13. Naylor A. Intervertebral disc prolapse and degeneration The biochemical and biophysical approach. Spine 1976, 1:108-114.

12. Wood G II, Lower back pain and disorders of intervertebral disc. In Canale T (ed) Campbell's Operative Orthopaedics. Mosby, Washington DC, 1998; pp 3014-3080.

14. DePalma AF and Rothman RH. Lumbar disc lesions: anatomic and pathologic features and the clinical syndrome. In DePal- ma AF, Rothman RH (eds). The Intervertebral Disc. WB Saunders Company, Philadelphia, 1970; pp 58-84.

15. Miller JAA, Schmatz C, Schultz AB. Lumbar disc degeneration: correlation with age, sex and spine level in 600 autopsy specimen. Spine 1988; 13:2173

16. Bobest $\mathrm{M}$, Furo I, Tompa $\mathrm{K}$ et al. $1 \mathrm{H}$ nuclear magnetic resonance study of intervertebral discs: a preliminary report. Spine 1986; 11:709-711.

17. Gibson MJ, Szypryt EP, Buckley JH et al. Magnetic resonance imaging of adolescent disc herniation. J Bone J Surg 1987;69-B:699.

18. Birney TJ, White JJ, Berens D et al. Comparison of MRI and discography in the diagnosis of lumbar degenerative disc disease. $J$ Spinal Disord 1992; 5:417-423.

19. Lindblom K. Diagnostic puncture of intervertebral disks in sciatica. Acta Orthop Scand 1948; 17: 213-239.

20. Colhoun E, McCall I, Williams L et al. Provocation discography as a guide to planning operations on the spine. J Bone / Surg [Br] 1988; 70: 267-271.

21. Hartman J, Kendrick J, Larman P. Discography as an aid in evaluation for lumbar and lumbosacral fusion. Clin Orthop 1977; 81: 77-81.

22. White A III and Panjabi M. Physical properties and functional biomechanics of the spine. In White A III, Panjabi M (eds). Clinical Biomechanics of the Spine. JB Lippincott Company, Philadelphia,1990; pp 318

23. Hedman TP, Kostuik JP, Fernie GR et al. Design of an intervertebral disc prosthesis. Spine 1991;16: S256-260.

24. Hou TS, Tu Kai Y, Xu Y et al. Lumbar Intervertebral Disc Prosthesis; An experimental Study. Chinese Med J 1991; 104 : 381-386.

25. Kotani Y, Abumi K, Shikinami Y et al. Artificial intervertebral disc replacement using bioactive three-dimensional fabric: design, development, and preliminary animal study. Spine 2002; 27:929-935.

26. Peary MJ. Stereo radiography of lumbar spine motion. Acta Ortho Scan (Suppl) 1985;212:1-41.

27. Seligman JV, Gertzbein, Tile M et al. Computer analysis of spinal segment motion in degenerative disc disease with or without axial loading. Spine 1984;9:566-573.

28. Bao Qi-Bin and Yuan HA. Artificial disc technology. Neurosurgical Focus 2000;9: $1-7$

29. Lemaire JP, Skalli W, Lavaste $F$ et al. Intervertebral disc prosthesis. Results and prospects for the year 2000. Clin Orthop 1997; 337:64-76.

30. Bao QB, McCullen GM, Higham PH et al. The artificial disc; theory, design and materials. Biomaterials 1996; 17:1157-67.

31. Stefee A. Artificial disc elastomer core between flat rigid plate. US patent no 5,071,437, 1991.

32. Lee CK, Langrana NA, Parsons et al. Development of functional disc spacer (Artificial Disc) In Mayer, Weigel (Eds): The Artificial Disc, Heidelberg, Springer-Verlag 1991;pp79-83.

33. Edeland HG. Some additional suggestions for an intervertebral disc prosthesis. J Biomed Eng 1985; 7:57-62.

34. Edeland HG. Suggestions for a total elas to-dynamic intervertebral disc prosthesis. Biomater Med Devices Artif Organs 1981; 9:65-72.

35. Butner-Janz K, Helisch HJ, Schellnack K, et al. Intervertebral endoprosthesis. US Patent 4,759,766, 26 July 1988

36. Griffith SL, Shelokov AP, Buttner-Janz K et al. A multicenter retrospective study of the clinical results of the LINK SB Charite intervertebral prosthesis. The initial European experience Spine 1994;19:1842-1849.

37. Cinotti G, David T, Postacchini F. Results of disc prosthesis after a minimum followup period of 2 years Spine 1996; 21:9951000.

38. Zeegers WS, Bohnen LM, Laaper $M$ et al. Artificial disc replacement with the modular type SB Charite III: 2-year results in 50 prospectively studied patients. Eur Spine 1999;8:210-217.

39. Enker P, Steffee A, Mcmillin C et al. Artificial disc replacement. Preliminary report with a 3-year minimum follow-up. Spine 1993;18:1061-1070.

40. Pointillart V. Cervical disc prosthesis in humans: first failure Spine 2001;1;26:E90E92.

41. Bao QB, Yuan HA. Prosthetic disc replacement: the future? Clin Orthop 2002;394: 139-145. 